\title{
Red Cell Distribution Width with CHADS2 and CHA2DS2-VASc score is associated with Post-operative Atrial Fibrillation after Coronary Artery Bypass Grafting
}

\author{
Alev Kılıcgedik, MD, Abdulrahman Naser, MD, Ahmet Seyfeddin Gurbuz, MD, \\ Seyhmuz Kulahcioglu, MD, Ruken Bengi Bakal, MD, Tuba Unkun, MD, Fatih Yilmaz, MD, \\ Gokhan Kahveci, MD, Cevat Kirma, MD
}

Kartal Koşuyolu Heart \&Research Hospital, Department of Cardiology, Istanbul, Turkey

\section{ABSTRACT}

Background: The use of the CHA2DS2-VASc scoring system and red cell distribution width (RDW) as post-op Atrial Fibrillation (POAF) predictors may be promising for the identification of patients that are at a higher risk of POAF.

Methods: A total of 358 patients (57 patients with POAF, and 301 patients with non-POAF ) with sinus rhythm undergoing a coronary artery bypass graft (CABG) operation were included in the study retrospectively. Preoperative RDW levels and electrocardiograms with sinus rhythm were recorded. Patients with at least one 12-lead electrocardiogram with atrial fibrillation in the postoperative period, with or without medical or electrical cardioversion, were considered to have postoperative atrial fibrillation. A CHADS2 and CHA2DS2-VASc score was calculated for all of the patients.

Results: RDW levels were significantly higher in POAF group. RDW levels were significantly correlated with CHADS2 $(r=0.15, P=.007)$ and CHA2DS2-VASc $(r=0.19$ $P=.0001)$ scores. CHA2DS2-VASc scores were significantly higher in patients with POAF, whereas CHADS2 scores did not differ between groups. In multivariate analysis, left atrial diameter (LAD) (OR:2.44 [95\% CI $1.16-5.1], P=.018)$, age (OR:1.04 [95\% CI $1.01-1.08$ ] $P=.01)$, and RDW (OR:1.16 [95\% CI $1.0-1.36], P=.05$ ) were found to be predictive for POAF. The area under the receiver-operating characteristic curve of RDW was $0.65(0.57-0.72, P=.0001)$ with $68.4 \%$ sensitivity and $51.2 \%$ specificity to predict POAF.

Conclusion: Our study showed that age, LAD, and the reduced probability of RDW are predictors of POAF, and that $\mathrm{RDW}$ is strongly associated with the thromboembolic risk as determined by CHADS2 and CHA2DS2-VASc scores.

\section{INTRODUCTION}

Post-operative atrial fibrillation (POAF) is the most common arrhythmia after coronary artery bypass grafting

Received August 9, 2017; received in revised form December 1, 2017; accepted fanuary 25, 2018.

Correspondance: Alev Klincgedik, MD, Kartal Koşuyolu Heart \&Research Hospital, Cardiology Clinic, 34846, Kartal, Ïstanbul, TR; 90216-500-1-500, fax:0216-4596321 (e-mail: akilicgedik@yahoo.com).
(CABG) operation, and affects $10 \%$ to $40 \%$ of patients undergoing CABG [Anatolevna 2016; Bohatch 2015]. This arrhythmia is associated with higher short and long term mortality rates, as well as higher prevelance of post-operative complications, including stroke, pneumonia, respiratory failure, and longer hospitalization [Folla 2016; Bohatch 2015; Mansoor 2014; Villareal 2004]. The exact mechanism of POAF is not fully understood, and a method of risk classification has not been established. Use of the CHA2DS2-VASc score (congestive heart failure; hypertension; age $>75$ years [doubled]; type 2 diabetes; previous stroke, transient ischemic attack [TIA], or thromboembolism [doubled]; vascular disease; age 65-75 years; and sex category), which extends the CHADS2 score (congestive heart failure, hypertension, age $>75$ years, type 2 diabetes, and previous stroke or TIA [doubled]) by considering additional stroke risk factors, was recently recommended to guide antithrombotic therapy in patients with atrial fibrillation (AF) or atrial flutter [European Heart Rhythm Association 2010]. Each of these components has been associated with the ventricular remodeling, left ventricular (LV) diastolic dysfunction, and left atrial enlargement, which may cause the atrial fibrillation [Chua 2013].

The red blood cell distribution width (RDW) is a rather simple measure of red blood cell (RBC) size heterogeneity. RDW has an important role in many acute and chronic cardiovascular diseases, including acute coronary syndrome (ACS), ischemic cerebrovascular disease, peripheral artery disease (PAD), heart failure (HF), AF, and hypertension, which are often associated with a high degree of anisocytosis. The association between RDW and non-valvular or paroxysmal AF with or without CHA2DS2-VASc score has been shown in some studies [Lui 2017; Lee 2015; Kurt 2014]. Moreover, baseline RDW levels were found to be associated with POAF [Korantzopoulos 2015; Ertaş 2013]. However, these studies have not had a strong enough impact to change treatment algorithms.

The use of the CHA2DS2-VASc scoring system and RDW as POAF predictors may be promising for the identification of patients that are at a higher risk of POAF. In this study, we investigated the relationship of RDW and CHA2DS2-VASc score in patients with POAF after the early post-CABG period. 
Table 1. Demographic, Clinical and Echocardiographic Characteristics of the Study Population

\begin{tabular}{lccc}
\hline & POAF & non-POAF & $P$ \\
\hline Age & $65.2 \pm 8.8$ & $60.5 \pm 9.5$ & .001 \\
Gender (F, \%) & $14(24.6 \%)$ & $52(17.4 \%)$ & .20 \\
BMI & $28.08 \pm 3.0$ & $28.02 \pm 3.5$ & .90 \\
DM (n, \%) & $23(40.4 \%)$ & $124(41.2 \%)$ & .90 \\
HT (n, \%) & $35(61.4 \%)$ & $170(56.9 \%)$ & .52 \\
HL (n, \%) & $48(87.3 \%)$ & $262(92.9 \%)$ & .15 \\
CHADS2 & $1.29 \pm 0.82$ & $1.16 \pm 0.86$ & .30 \\
CHA2DS2-VASc & $3.03 \pm 1.05$ & $2.68 \pm 1.14$ & .034 \\
ACS (n, \%) & $30(52.6 \%)$ & $164(45.5 \%)$ & .79 \\
Stable CAD (n, \%) & $27(47.4 \%)$ & $137(54.5 \%)$ & .79 \\
Beta-blocker (n, \%) & $53(93 \%)$ & $289(96 \%)$ & .31 \\
ACEI & $28(49.1 \%)$ & $137(45.5 \%)$ & .61 \\
LVEF (\%) & $55.9 \pm 10.5$ & $56 \pm 10.1$ & .9 \\
LAD (cm) & $3.8 \pm 0.43$ & $3.6 \pm 0.36$ & .003 \\
LVEDD (cm) & $4.9 \pm 0.78$ & $4.8 \pm 0.52$ & .54 \\
LVESD (cm) & $3.3 \pm 0.72$ & $3.3 \pm 1.6$ & .88 \\
E/A < 1 (n, \%) & $37(64.9 \%)$ & $210(69.8 \%)$ & .09 \\
\hline
\end{tabular}

BMI indicates body mass index; DM, diabetes mellitus; $H T$, hypertension; $\mathrm{HL}$, hyperlipidemia; ACS, acute coronary disease; CAD, coronary artery disease; ACEI, ace inhibitor; LVEF, left ventricular ejection fraction; LAD, left atrial diameter; LVEDD, left ventricular end-diastolic diameter; LVESD, left ventricular end-systolic diameter.

\section{METHODS}

This study was approved by a local ethics committee. A total of 358 patients (57 patients with POAF, and 301 patients with non-POAF ) undergoing a CABG operation in our hospital between January 2016 and March 2017 were included in the study retrospectively. Patients over 18 years of age with sinus rhythm were included in the study consecutively. All data were provided by the patients' hospital files and the computer data system of our hospital. Patients with previous atrial arrhythmia, active infections, significant anemia and renal failure, and concomitant valve surgery were excluded from the study. There were no chronic inflammatory diseases reported for any patients.

Preoperative hematological data including complete blood count, biochemical investigations, and coventional inflamatory indexes such as white blood cell (WBC), C-reactive protein (CRP), and electrocardiograms (ECG) with sinus rhythm were recorded. Patients that had at least one 12-lead electrocardiogram with atrial fibrillation in the first three postoperative days, with or without medical or electrical cardioversion, were considered as having postoperative atrial fibrillation. All patients were given a 12-lead ECG within the 24 hours before, and the 72 hours following, the CABG operation. All patients
Table 2. Labaratory Findings of The Study Population

\begin{tabular}{lccc}
\hline & POAF & non- POAF & $P$ \\
\hline WBC & $8,18 \pm 1.94$ & $8.34 \pm 2.29$ & .62 \\
HGB & $12.8 \pm 1.73$ & $13.4 \pm 1.78$ & .029 \\
PLT & $249.2 \pm 69.2$ & $231.3 \pm 68.2$ & .07 \\
RDW & $14.6 \pm 2.04$ & $13.9 \pm 1.4$ & .002 \\
CRP & $4.98 \pm 22.1$ & $2.22 \pm 8.2$ & .105 \\
NLR & $3.0 \pm 1.3$ & $2.8 \pm 1.77$ & .41 \\
Sedimentation & $32.6 \pm 25.1$ & $33.7 \pm 27.1$ & .78 \\
UREA & $41.1 \pm 12.0$ & $40.5 \pm 16.8$ & .8 \\
CREA & $0.90 \pm 0.25$ & $0.88 \pm 0.23$ & .52 \\
UA & $6.08 \pm 1.5$ & $6.8 \pm 8.5$ & .53 \\
Na & $137.2 \pm 3.1$ & $135.5 \pm 13.6$ & .35 \\
K & $4.3 \pm 0.48$ & $4.3 \pm 0.43$ & .87 \\
Total cholesterol & $194.0 \pm 51.6$ & $201.2 \pm 55.8$ & .37 \\
HDL & $40.9 \pm 8.9$ & $40.1 \pm 8.6$ & .51 \\
LDL & $113.0 \pm 39.8$ & $122.2 \pm 42.1$ & .15 \\
Triglyceride & $195.9 \pm 119.3$ & $205.9 \pm 208.6$ & .73 \\
\hline
\end{tabular}

NLR indicates Neutrophil Lymphocyte Ratio; WBC, white blood cell; HGB, hemoglobin; PLT, platelets; RDW, red cell distribution width; CRP, C-reactive protein; CREA, creatine; UA, uricacid; HDL, high-density lipoprotein; LDL, low-density lipoprotein.

were monitored for 72 hours, and patients who developed AF during monitoring were also given a 12-lead ECG. All electrocardiograms were analyzed by an expert cardiologist.

The CHADS2 score was calculated for all the patients by assigning one point for each of the following criteria: congestive heart failure, hypertension, age of at least 75 years, and diabetes mellitus. For the criterion of previous stroke or TIA, two points were added. In contrast, the CHA2DS2-VASc score is based on a point system in which two points each are assigned for an age of at least 75 years and history of stroke/TIA/thromboembolism, and one point is assigned for each of the following criteria: congestive heart failure, hypertension, diabetes mellitus, age of 65 to 75 years, female sex category, and vascular disease (defined as previous myocardial infarction, complex aortic plaque, carotid disease, and peripheral arterial disease $[\mathrm{PAD}]$, including intermittent claudication, previous surgery or percutaneous intervention on the abdominal aorta or the lower extremity vessels, and arterial and venous thrombosis).

Before CABG operation, all patients underwent a transthoracic 2-dimensional and Doppler echocardiographic assessment, using a 1 to $5 \mathrm{MHz}$ S5-1 transducer (iE33, Philips Healthcare, Inc., Andover, MA). Patients were examined in the left lateral position, and measurements were averaged over three consecutive heart cycles. All 
standard 2D transthoracic echocardographic images from parasternal long axis, short axis, apical four, three, and two chamber views, and color Doppler images were stored in cine-loop format, triggered to the QRS complex. The $\mathrm{LV}$ diastolic and systolic diameters were measured using M-mode or 2-dimensional echocardiography. Left ventricular ejection fraction (LVEF) was calculated according to Simpson's formula, employing a two-dimensional image of the LV chamber during systole and diastole in the four and two chamber apical views.

\section{STATISTICAL ANALYSIS}

Statistical analyses were performed using SPSS 17.0 (Chicago). The Kolmogorov-Smirnov test was used to analyze normality of the data. Continuous data were expressed as mean \pm standard deviation (SD), and categorical data were expressed as percentages. The chi-square test was used to assess differences in categorical variables between groups. The relationships among parameters were assessed using Pearson's or Spearman's correlation analysis, according to the normality of the data. Differences between groups were tested using Mann-Whitney U or Student's T testing as appropriate. Receiver-operating characteristic (ROC) curves were constructed to determine the optimum cut-off for the RDW value that best predicted post-operative atrial fibrillation. The area under the curve (AUC) was calculated to quantify sensitivity and specificity. The logistic regression analysis was examined to determinate the relationship between independent variables. A $P$-value $<.05$ was considered statistically significant.

\section{RESULTS}

The patients' demographic and echocardiographic findings are shown in Table 1. The incidence of POAF was $15.9 \%$ in our patient group. The mean age of all patients was 61.3 \pm 9.5 years, and was higher in the POAF group $(65.2 \pm 8.8$ in POAF group and $60.5 \pm 9.5$ in non-POAF group, $P=.001)$. There was no statistical difference between the POAF and non-POAF groups in terms of gender $(P=.20)$, body mass index $(\mathrm{BMI})(P=.90)$, diabetes mellitus $(\mathrm{DM})(P=.90)$, hypertension (HT) $(P=.52)$, or hyperlipidemia $(\mathrm{HL})(P=.15)$.

The diagnosis given during admission to the hospital (acute coronary syndrome and stable coronary artery disease) did not differ between groups $(P=.79)$.

CHA2DS2-VASc scores were significantly higher in patients with POAF, though CHADS2 scores did not differ.

Although LVEF, LV end diastolic diameter (LVEDD), and LV end systolic diameter (LVESD) were not statistically different between patients of both groups, the left atrial diameter (LAD) was higher in POAF group than in non-POAF group $(3.8 \pm 0.43$ and $3.6 \pm 0.36$, respectively, $P=.003)$. $\mathrm{E} / \mathrm{A}<1$ ratios were used as an indicator of diastolic dysfunction, and were not different among groups.

The laboratory findings of the patients are shown in Table 2. Hemoglobin levels were lower in the POAF group, and the RDW levels were significantly higher (Figure 1).

RDW was positively correlated with age $(r=0.192$,

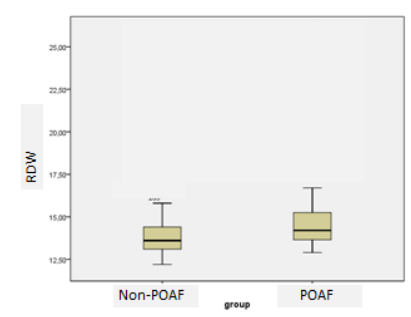

Figure 1. Box-plot of RDW levels in POAF and non-POAF groups.
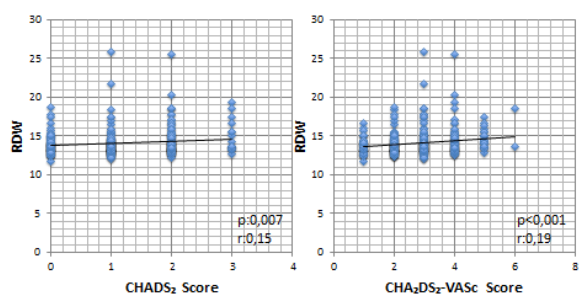

Figure 2. Correlations between RDW and CHADS2 and CHA2DS2VASc scores.

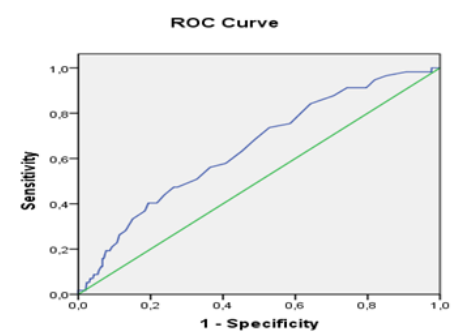

Figure 3. The area under the receiver-operating characteristic (ROC) curve of RDW was $0.65(0.57-0.72, P=.0001)$, with $68.4 \%$ sensitivity and $51.2 \%$ spesificity to predict POAF.

$P=.0001), \operatorname{LAD}(\mathrm{r}=0.166, P=.002)$, and urea $(\mathrm{r}=0.151$, $P=.004)$, and negatively correlated with hemoglobin levels ( $\mathrm{r}=-0.183, P=.001)$.

CHA2DS2-VASc score was also significantly correlated with LAD $(\mathrm{r}=0.138, P=.012)$, and negatively correlated with LVEF $(\mathrm{r}=-0.109 . P=.043)$ and hemoglobin $(\mathrm{r}=-.0 .26, P=.0001)$.

There was a significant correlation between RDW and CHADS2 scores $(r=0.15, P=.007)$, and RDW and CHA2DS2-VASc scores $(\mathrm{r}=0.19, P=.0001)$ (Figure 2$)$.

In multivariate analysis, LAD (OR:2.44 [95\% CI 1.16 - 5.1], $P=.018)$, age (OR:1.04 [95\% CI 1.01 - 1.08], $P=.01)$, and less probable RDW (OR:1.16 [95\% CI 1.0 - 1.36], $P=.05$ ) were found to be predictive for POAF (Figure 3 ). The area under the ROC curve of RDW was 0.65 (0.57 - 0.72 , $P=.0001)$ with $68.4 \%$ sensitivity and $51.2 \%$ spesificity to predict POAF (Figure 4).

\section{DISCUSSION}

Our study is consistent with other studies that demonstrated that the LAD and age were the main predictors of 
POAF, and that RDW tended to predict POAF [Folla 2016; Korantzopoulos 2015; Ertaş 2013]. Advancing age is the most prominent risk factor for AF; degenerative changes such as atrial fibrosis and dilatation lead to changes in the electrical characteristics of the sinus and AV nodes [Staerk 2017; Yadava 2016; Bohatch 2015]. Villereal et al found that an age greater than 65 years was an independent risk factor for development of POAF after revascularization [Villareal 2004]. It is well established that atrial enlargement and stretch facilitate the occurence of $\mathrm{AF}$ by increasing atrial vulnerability [Chua 2013]. Moreover, Gabrielli et al found that left atrial dysfunction, evaluated by strain and strain rate, is an independent predictor of POAF and contributes to classical risk factors like age and atrial volume [Gabrielli 2011].

Although RDW is not a direct measure of anisocytosis, it is an easy measure of red blood cell (RBC) size heterogeneity within the complete blood cell count. AF, like many cardiovascular diseases, is often associated with a high degree of anisocytosis because of oxidative stress and chronic inflammation [Danese 2015]. RDW and changes in RDW were found to be independently associated with the risk of all causes of mortality in patients with AF [Saliba 2017]. In a pilot observatonal study of 109 patients, Korantzopoulos et al showed that baseline RDW levels were associated with the occurence of POAF after elective cardiac surgery [Korantzopoulos 2015]. Consistent with this study, Ertaş et al showed that preoperative RDW level predicted new-onset $\mathrm{AF}$ after CABG [Ertaş 2013]. In our study, RDW was found to be significantly higher in POAF group, however, in multivariate analysis it was not found to be as significant as in the aforementioned studies.

The RDW and CHA2DS2-VASc scores were found to be significantly higher in the POAF group, though CHADS2 scores did not differ between the patients of the two groups. Although Chua et al showed that CHADS2 and CHA2DS2VASc scores could be helpful, convenient scoring systems for predicting POAF after cardiac surgery, in our study group, CHADS2 and CHA2DS2-VASc scores were not significant predictors of POAF in multivariate analysis [Chua 2013]. However, RDW was found to be significantly correlated with CHADS2 and CHA2DS2-VASc scores. Some studies $(8,10)$ have shown that RDW values are significantly correlated with CHA2DS2-VASc scores in nonanemic, non-valvular $\mathrm{AF}$ patients [Kurt 2014]. This is not, in fact, an unexpected result, because RDW was revealed to be associated with and higher in some components of CHADS2 and CHA2DS2VASc scores, such as heart failure, stroke, coronary heart disease, HT, and DM [Makhoul 2013; Tanindi 2012; Azab 2011; Sánchez-Chaparro 2010; Ani 2009]. RDW was also found to be associated with AF itself [Saliba 2017; Lee 2015; Güngör 2014]. It has also been shown to be a strong independent predictor of morbidity and mortality in acute decompansated heart failure [Makhoul 2013]. Increased levels of RDW are associated with stroke, and are predictive for all causes of deaths in patients with a known history of stroke [Ani 2009]. RDW also has predictive value in non-STMI patients for all-cause long-term mortality [Azab 2011]. In addition, higher RDW values are correlated with higher systolic and diastolic blood pressures and metabolic syndrome [Tanindi 2012; Sánchez-Chaparro 2010]. In our study, we demonstrated that these scoring systems may be usable in, and associted with, POAF as well.

Although each component of the CHADS2 and CHA2DS2-VASc scores has been associated with the ventricular remodeling, LV diastolic function, and LA enlargement, in our study, there was no significant difference in LVEF, LVEDD, LVESD, or diastolic dysfunction between the patients of the two groups [Chua 2013]. However, LAD was shown to be significantly higher in the POAF group. Despite the fact that some studies found LVEF as a predictor of POAF, and found an association between $\mathrm{LV}$ diastolic dysfunction and an increased incidence of POAF, these were not confirmed in our study [Onk 2015; Ashes 2014].

In the literature, an exagerated inflamatory response was shown to be associated with a higher risk of POAF, but we were unable to find a correlation between systemic inflamation and POAF among the patients in our study [Aviles 2003]. There was no significant difference in baseline CRP levels, Neutrophil Lymphocyte Ratio (NLR), or WBC counts between POAF and non-POAF group. In contrast with this finding, the study of Şaşkın et al showed that elevated preoperative CRP levels were associated with development of POAF in a relatively large cohort [Şaşkın 2016].

\section{LIMITATIONS}

This is a retrospective study; all information about the patients were provided from their files. Some factors such as iron, vitamin B12, and folic acid levels, which can influence RDW levels, were not determined in our study. No followup data is available to clarify the relation between RDW and thromboembolic events. Further prospective studies with large numbers of patients are required in order to clarify the predictive value of RDW in POAF patients.

\section{CONCLUSION}

Our study showed that age, LAD, and a lower probability of RDW are the predictors of POAF. RDW was also demonstrated to be strongly associated with thromboembolic risk, as determined by CHADS2 and CHA2DS2-VASc scores. The use of RDW with CHADS2 and CHA2DS2-VASc scores may be helpful as a simple method for better identification of patients that are at higher risk of POAF and thromboembolism. However, to assess the value of RDW as a predictor of thromboembolic events, further prospective studies with large number of patients are required.

\section{REFERENCES}

Anatolevna RO, Veniaminovich FO, Mikhaylovich KS. 2016. Predictors of new-onset atrial fibrillation in elderly patients with coronary artery disease after coronary artery bypass graft. J Geriatr Cardiol 13:444-9.

Ani C, Ovbiagele B. 2009. Elevated red blood cell distribution width 
predicts mortality in persons with known stroke. J Neurol Sci 277:103-8. Ashes CM, Yu M, Meineri M, et al. 2014. Diastolic dysfunction, cardiopulmonary bypass, and atrial fibrillation after coronary artery bypass graft surgery. Br J Anaesth 113:815-21.

Aviles RJ, Martin DO, Apperson-Hansen C, et al. 2003. Inflammation as a risk factor for atrial fibrillation. Circulation 108:3006-10.

Azab B, Torbey E, Hatoum H, et al. 2011. Usefulness of red cell distribution width in predicting all-cause long-term mortality after non-STelevation myocardial infarction. Cardiology 119:72-80.

Bohatch Júnior MS, Matkovski PD, Di Giovanni FJ, et al. 2015. Incidence of postoperative atrial fibrillation in patients undergoing on-pump and off-pump coronary artery bypass grafting. Rev Bras Cir Cardiovasc 30:316-24.

Chua SK, Shyu KG, Lu MJ, et al. 2013. Clinical utility of CHADS2 and CHA2DS2-VASc scoring systems for predicting postoperative atrial fibrillation after cardiac surgery. J Thorac Cardiovasc Surg 146:919-26.

Danese E, Lippi G, Montagnana M. 2015. Red blood cell distribution width and cardiovascular diseases. J Thorac Dis 7:E402-11.

Ertaş G, Aydin C, Sönmez O, et al. 2013. Red cell distribution width predicts new-onset atrial fibrillation after coronary artery bypass grafting. Scand Cardiovasc J 47:132-5.

European Heart Rhythm Association, European Association for CardioThoracic Surgery, Camm AJ, et al. 2010. Guidelines for the management of atrial fibrillation: the task force for the management of atrial fibrillation of the european society of cardiology (ESC). Eur Heart J 31:2369-429.

Folla CO, Melo CC, Silva RC. 2016. Predictive factors of atrial fibrillation after coronary artery bypass grafting. Einstein (Sao Paulo) 14:480-5.

Gabrielli L, Corbalan R, Córdova S, et al. 2011. Left atrial dysfunction is a predictor of postcoronary artery bypass atrial fibrillation: association of left atrial strain and strain rate assessed by speckle tracking. Echocardiography 28:1104-8.

Güngör B, Özcan KS, Erdinler İ, et al. 2014. Elevated levels of RDW is associated with non-valvular atrial fibrillation. J Thromb Thrombolysis 37:404-10.

Korantzopoulos P, Sontis N, Liu T, et al. 2015. Association between red blood cell distribution width and postoperative atrial fibrillation after cardiac surgery: a pilot observational study. Int J Cardiol 185:19-21.
Kurt M, Tanboga IH, Buyukkaya E, et al. 2014. Relation of red cell distribution width with CHA2DS2-VASc score in patients with nonvalvular atrial fibrillation. Clin Appl Thromb Hemost 20:687-92.

Lee KH, Park HW, Cho JG, et al. 2015. Red cell distribution width as a novel predictor for clinical outcomes in patients with paroxysmal atrial fibrillation. Europace 17 Suppl 2:ii83-8.

Liu T, Shao Q, Korantzopoulos P et al. 2017. Relation of red blood cell distribution width with CHADS2 and CHA2DS2-VASc score in Chinese patients with non-valvular atrial fibrillation. Int J Cardiol 228:861-864.

Makhoul BF, Khourieh A, Kaplan M, et al. 2013. Relation between changes in red cell distribution width and clinical outcomes in acute decompensated heart failure. Int J Cardiol 167:1412-6.

Mansoor E. 2014. De novo atrial fibrillation post cardiac surgery: the Durban experience. Cardiovasc J Afr 25:282-7.

Onk OA, Erkut B. 2015. Is the preoperative administration of amiodarone or metoprolol more effective in reducing atrial fibrillation: after coronary bypass surgery? Medicine (Baltimore). 94:e1576.

Saliba W, Barnett-Griness O, Rennert G. 2017. Red cell distribution width and all-cause mortality in patients with atrial fibrillation: a cohort study. J Arrhythm 33:56-62.

Sánchez-Chaparro MA, Calvo-Bonacho E, González-Quintela A, et al 2010. Higher red blood cell distribution width is associated with the metabolic syndrome: results of the ibermutuamur cardiovascular risk assessment study. Diabetes Care 33:e40.

Şaşkın H, Düzyol Ç, Aksoy R, et al. 2016. Do preoperative C-reactive protein and mean platelet volume levels predict development of postoperative atrial fibrillation in patients undergoing isolated coronary artery bypass grafting? Postepy Kardiol Interwencyjnej 12:156-63.

Staerk L, Sherer JA, Ko D, et al. 2017. Atrial fibrillation: epidemiology, pathophysiology, and clinical outcomes. Circ Res 120:1501-17.

Tanindi A, Topal FE, Topal F, et al. 2012. Red cell distribution width in patients with prehypertension and hypertension.Blood Press 21:177-81.

Villareal RP, Hariharan R, Liu BC, et al. 2004. Postoperative atrial fibrillation and mortality after coronary artery bypass surgery. J Am Coll Cardiol 43:742-8.

Yadava M, Hughey AB, Crawford TC. 2016. Postoperative atrial fibrillation: incidence, mechanisms, and clinical correlates. Heart Fail Clin 12:299-308. 